\title{
ATENDIMENTO ODONTOLÓGICO DE PACIENTE SUBMETIDO À RADIOTERAPIA EM REGIÃO DE CABEÇA E PESCOÇO: RELATO DE CASO CLÍNICO
}

\author{
Bianca Segantini BORGES 1 \\ e-mail: bianca.segantini@hotmail.com
}

\author{
Daniela Assis do VALE \\ e-mail:dani.asssis@usp.br
}

Renata $\mathrm{AOKI}^{3}$

e-mail:aokireanta@hotmail.com

Tarcila TRIVINO ${ }^{4}$

e-mail:tarcilatrivino@uol.com.br

\author{
Karin Sá FERNANDES 5
}

e-mail: karin.fernandes@usp.br

\section{RESUMO}

O tratamento do câncer de cabeça e pescoço depende da idade do paciente, presença de comorbidades, tamanho do tumor, localização, grau, estágio, presença de linfonodos afetados e de metástases, e inclui cirurgia, radioterapia e quimioterapia, combinadas ou separadamente. As complicações bucais da radioterapia em região de cabeça e pescoço são amplamente conhecidas, tais como, mucosite, xerostomia, disgeusia, trismo, cárie de radiação e osteorradionecrose. A prevenção ou redução da incidência e severidade dessas complicações são fundamentais para a manutenção da saúde bucal, tendo o dentista um papel primordial antes, durante e após a radioterapia. Dessa forma, este relato de caso visa discutir o atendimento odontológico de uma paciente irradiada em região de cabeça e pescoço, salientando as principais complicações bucais, manejo odontológico e a importância da Odontologia na equipe multidisciplinar no tratamento do câncer bucal.

DESCRITORES: CARCINOMA DE CÉLULAS ESCAMOSAS; RADIOTERAPIA; ODONTOLOGIA; MUCOSITE UNIVERSIDADE CIDADE DE SÃO PAULO - UNICID

\footnotetext{
Aluna de graduação da Universidade Cidade de São Paulo - UNICID

Professora de graduação da Universidade Cidade de São Paulo - UNICID

Aluna do Mestrado Profissionalizante da Universidade Cidade de São Paulo - UNICID

Professor de graduação e pós-graduação Universidade Cidade de São Paulo - UNICID

Professor de graduação e pós-graduação Universidade Cidade de São Paulo - UNICID
} 


\title{
DENTAL CARE OF PATIENT SUBMITTED TO RADIOTHERAPY IN THE HEAD AND NECK REGION: A CASE REPORT
}

\begin{abstract}
The head and neck cancer treatment depend on age, comorbidities, tumor size, site, stage, grade, lymph nodes status and metastases, and include surgery, radiotherapy and chemotherapy, combined or separately. Oral complications of radiotherapy in the head and neck region are widely known, such as mucositis, xerostomia, dysgeusia, trismus, radiation caries and osteoradionecrosis. The prevention or reduction of incidence and severity of oral complications are essential in the maintenance of oral health, and the dentist having a primary role before, during and after radiotherapy. Thus, this case report aims to discuss the dental care of a patient irradiated in the head and neck region, highlighting the main oral complications, dental management and the importance of Dentistry in the multidisciplinary team in the treatment of oral cancer.
\end{abstract}

DESCRIPTORS : CARCINOMA, SQUAMOUS CELL; RADIOTHERAPY; DENTISTRY; MUCOSITIS

\section{INTRODUÇÃO}

As neoplasias malignas em cabeça e pescoço são a $6^{a}$ neoplasia maligna mais prevalente no mundo ${ }^{1,2}$. No Brasil foram estimados 14.700 novos casos de câncer em cavidade oral e orofaringe para 2018 pelo Instituto Nacional do Câncer ${ }^{3}$. Aproximadamente $90 \%$ dessas neoplasias são diagnosticadas como carcinoma epidermoide ou, na denominação antiga, carcinoma espinocelular (CEC) ${ }^{4,5}$. O tratamento do CEC depende especialmente do sítio da lesão, do tamanho do tumor, da presença de linfonodos afetados e de metástases, e inclui a cirurgia, radioterapia e quimioterapia, combinadas ou separadamente ${ }^{6}$.

A radioterapia é o tratamento mais utilizado no câncer de cabeça e pescoço, e consiste em radiações ionizantes, que atuam sobre o DNA das células malignas, levando à morte celular ou à perda de sua capacidade reprodutiva e, ao mesmo tempo, preserva os tecidos normais alcançando um índice terapêutico favorável. Porém, altas doses de radiação em extensos campos levam a efeitos desfavoráveis ao organismo, principalmente na mucosa oral?.

As alterações orais associadas à radioterapia são: mucosite, xerostomia, disgeusia, trismo, cárie por radiação e osteorradionecrose. Esses efeitos adversos podem afetar o tratamento oncológico, além de influenciar negativamente na qualidade de vida dos pacientes ${ }^{8}$.

O tratamento odontológico prévio à radioterapia é imprescindível, visando à prevenção e/ou diminuição dessas possíveis complicações. O dentista deve realizar exame clínico minucioso, avaliação óssea através de exames imaginológicos e promover, quando necessário, a remoção de focos infecciosos, através de tratamento periodontal, substituições de restaurações insatisfatórias, correções e substituições de próteses mal adaptadas e exodontias prévias ${ }^{9,10,11}$.

Durante a radioterapia, o dentista deve salientar a importância da saúde bucal, pois há predisposição a diversas infecções secundárias, principalmente devido à diminuição do fluxo salivar. É imprescindível a manutenção da higiene oral com dentifrícios fluoretados, uso de fio dental, bochechos com solução de fluoreto de sódio a 0,05\% e com clorexidina a 0,12\% $0^{9,10,11}$. 
BORGES BS, VALE DA, AOKI R, TRIVINO T, FERNANDES KS. Atendimento odontológico de paciente submetido à radioterapia em região de cabeça e pescoço: relato de caso clínico. Rev. Odontol. Univ. Cid. São Paulo 2018 jul/set 30(3) 332-40

ISSN 1983-5183

A primeira alteração que surge com o tratamento radioterápico é a disgeusia que ocorre pela atrofia das papilas gustativas causada pela radiação, associada à redução do fluxo salivar. A perda de paladar pode persistir por semanas ou meses,

A mucosite oral é outra manifestação bucal que se inicia aproximadamente 15 dias após o começo da radioterapia; consiste em um dano à mucosa oral e ao trato gastrointestinal, apresentando-se em graus variados de eritema e podendo evoluir para ulcerações. Uma das alternativas terapêuticas para a mucosite oral é a laserterapia de baixa potência, um tratamento não invasivo que promove rápida cicatrização e analgesia ${ }^{12,13}$.

A xerostomia ocorre devido à disfunção das glândulas salivares, induzindo o paciente à sensação de boca seca. Recomenda-se estimular o fluxo salivar por meio de gomas de mascar sem açúcar, uso de fluoretos (gel ou solução), reposição de líquidos e o uso de saliva artificial9,10.

O trismo é uma consequência observada tardiamente em pacientes submetidos à radioterapia, caracterizado pela limitação de abertura bucal. É causado pela exposição à radiação ionizante dos músculos masseter, temporal, pterigoides medial e lateral e da cápsula articular, levando o tecido à fibrose ${ }^{10}$. 0 tratamento deve começar quando o paciente apresentar os primeiros sinais clínicos de comprometimento de mobilidade de mandíbula, através de exercícios de fisioterapia para forçar a abertura de boca com adequada orientação ${ }^{8}$.

A candidose oral é uma infecção secundária que pode surgir devido a alterações na composição da saliva e diminuição do fluxo salivar. Apresenta-se como placas brancas destacadas facilmente, causando dor e queimação. $O$ tratamento é realizado com antifúngicos tópicos ou sistêmicos ${ }^{9,10}$.

Outra manifestação decorrente do tratamento radioterápico é a osteorradionecrose dos maxilares, que consiste em uma exposição de osso desvitalizado através de uma abertura na pele ou mucosa. 0 tratamento vai depender da presença ou ausência de inflamação e/ou infecção, sendo a terapia conservadora a mais indicada, através de irrigações com soluções antimicrobianas, administração de antibióticos e sequestrectomias ${ }^{8,9,13}$.

A cárie de radiação caracteriza-se por um tipo agressivo de cárie em indivíduos que receberam radioterapia em região de cabeça e pescoço, devido à redução significativa do fluxo salivar e às alterações dos constituintes salivares $8,10,14$.

Dessa forma, descrevemos um caso de um paciente irradiado em região de cabeça e pescoço, antes, durante e após a radioterapia, salientando as principais complicações bucais, manejo odontológico e a importância da Odontologia na equipe multidisciplinar no tratamento do câncer bucal.

\section{RELATO DE CASO CLÍNICO}

Paciente do sexo feminino, melanoderma, 60 anos de idade, com diagnóstico de CEC em orofaringe, compareceu ao consultório odontológico, encaminhada pelo serviço de radioterapia, sem queixas bucais, para adequação bucal prévia à radioterapia e quimioterapia. O tratamento oncológico instituído foi de 35 sessões de radioterapia conformacional (3D-RCT) em uma dose total de 70cGy, associada à quimioterapia.

Na anamnese, a paciente relatou ser ex-fumante e ex-etilista crônica. As principais comorbidades presentes eram hipertensão arterial e gastrite crônica, em uso diário de anti-hipertensivo, diurético, sinvastatina e omeprazol. 
BORGES BS, VALE DA, AOKI R, TRIVINO T, FERNANDES KS. Atendimento odontológico de paciente submetido à radioterapia em região de cabeça e pescoço: relato de caso clínico. Rev. Odontol. Univ. Cid. São Paulo 2018 jul/set 30(3) 332-40

\section{ISSN 1983-5183}

Ao exame clínico intrabucal prévio ao tratamento radioterápico, observou-se edentulismo superior, em uso de prótese total, e doença periodontal crônica nos dentes remanescentes do arco inferior (Figura1). Foi solicitada uma radiografia panorâmica, não sendo observados quaisquer sinais de anormalidades (Figura 2).
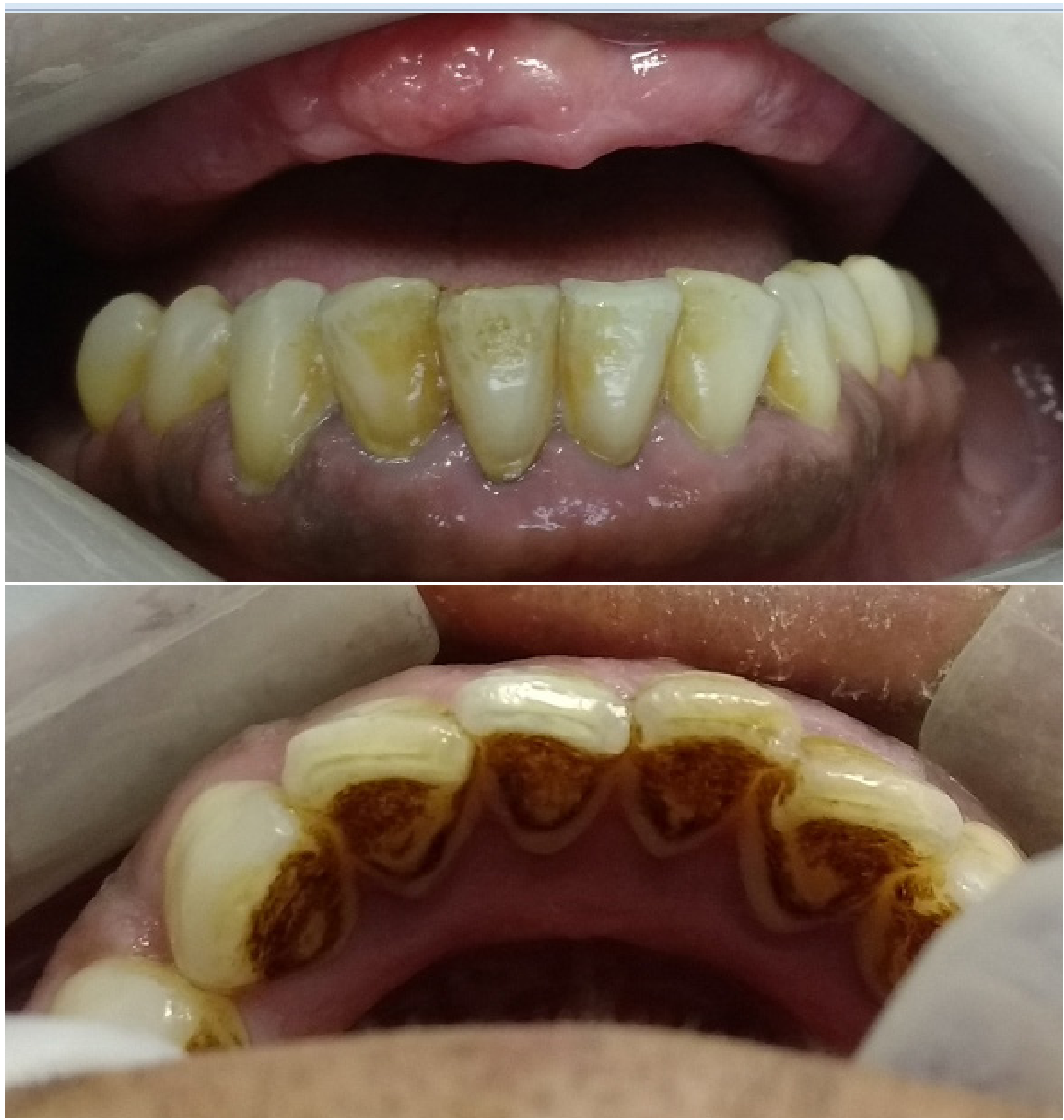

Figura 1 - Condição bucal prévia ao tratamento radioterápico: edentulismo superior, em uso de prótese total, e doença periodontal crônica nos dentes inferiores. 


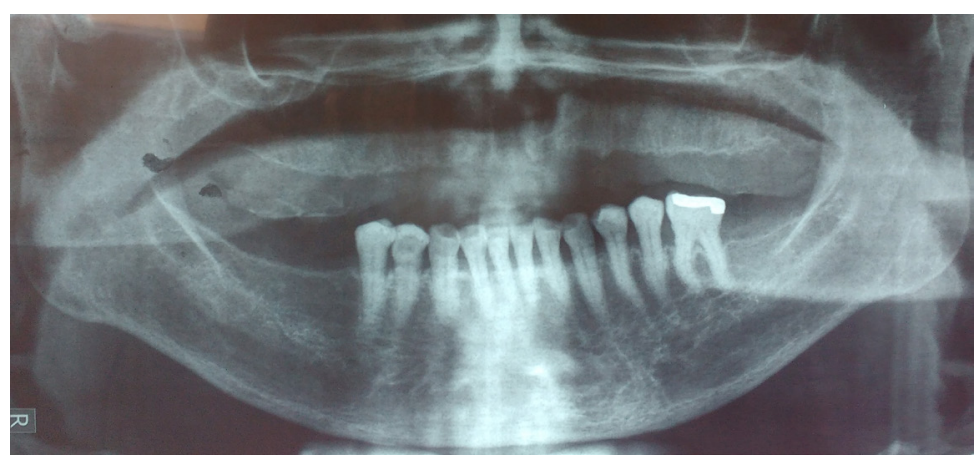

Figura 2 - Radiografia panorâmica pré-radioterapia.

O plano de tratamento proposto foi raspagem e alisamento corono-radicular, profilaxia dentária, orientação e motivação de higiene bucal, bochechos com clorexidina a 0,12\% por 7 dias, bochechos diários com NAF a 0,05\% e orientação sobre as complicações bucais e prevenção dos efeitos colaterais do tratamento antineoplásico.

Durante a terapia radioterápica, a paciente apresentou efeitos adversos na cavidade bucal, como: candidíase pseudomembranosa, xerostomia, perda de paladar e mucosite grau 4, segundo critério classificatório da Organização Mundial de Saúde (OMS) (Figura 3). Devido a esses efeitos, a paciente perdeu $11 \mathrm{~kg}$, sendo necessário o uso de sonda nasogástrica para alimentação e teve que interromper a radioterapia por um período de 2 semanas devido à debilidade física.

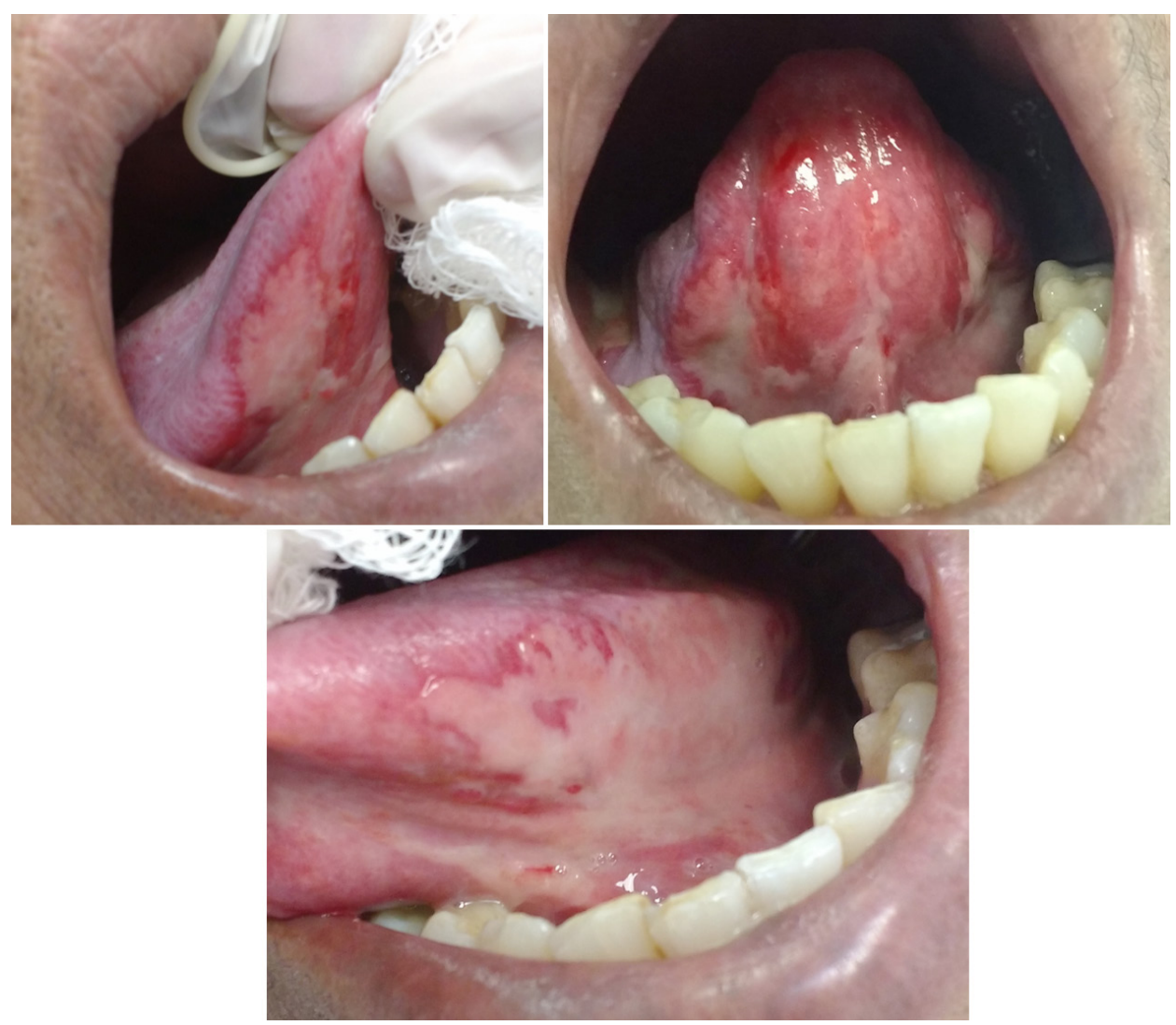

Figura 3 - Mucosite oral grau 4 após início da radioterapia. 
BORGES BS, VALE DA, AOKI R, TRIVINO T, FERNANDES KS. Atendimento odontológico de paciente submetido à radioterapia em região de cabeça e pescoço: relato de caso clínico. Rev. Odontol. Univ. Cid. São Paulo 2018 jul/set 30(3) 332-40

ISSN 1983-5183

Para o tratamento da mucosite, foi utilizado laser de baixa potência (GaA1As e InGaAIP), com comprimento de onde de $660 \mathrm{~nm}$, potência de $100 \mathrm{~mW}$ e energia de $2 \mathrm{~J}$, com aplicação pontual, no período de aparecimento das lesões bucais até 20 dias após o término da terapia antineoplásica. Para o tratamento da candidíase pseudomembranosa foram realizados bochechos com nistatina, e para a xerostomia, indicado o uso de lubrificantes bucal e labial.

Com o término do tratamento antineoplásico, a paciente encontra-se em acompanhamento odontológico trimestral, e não apresenta quaisquer focos de infecção bucal.

\section{DISCUSSÃO}

A presença do dentista na equipe multidisciplinar de tratamento a pacientes com câncer é fundamental para reduzir as complicações oriundas do tratamento antineoplásico, reduzir o tempo de internação e melhorar a qualidade de vida.

O acompanhamento odontológico pré-radioterapia é muito importante para os pacientes irradiados em região de cabeça e pescoço. Há muitos trabalhos que mostram redução da gravidade de mucosite oral em indivíduos que recebem adequação bucal prévia à irradiação ${ }^{10,14,15}$. Além disso, o risco de infecções bucais se reduz significativamente com a remoção dos focos infecciosos bucais antes da terapia radioterápica ${ }^{16}$.

Bueno et al. ${ }^{17}$ (2012) acompanharam 28 pacientes com câncer de cabeça e pescoço que iniciariam a radioterapia. Todos os pacientes receberam adequação bucal prévia à radioterapia, e foram monitorados semanalmente em relação a sua saúde bucal durante o tratamento antineoplásico, não sendo observado nenhum paciente com mucosite grau 4, e apenas $2(7,1 \%)$ indivíduos com mucosite grau 3. Os cuidados bucais se mostraram fundamentais para o controle de complicações agudas decorrentes da radioterapia.

Apesar de todos os cuidados bucais prévios à radioterapia, a paciente do referido caso clínico apresentou mucosite oral grau 4 (OMS), porém não foram observadas infecções bucais graves, apenas candidíase eritematosa em palato. Com o aparecimento da mucosite foi instituído o laser de baixa potência que trouxe grande melhora para a paciente em termos de alimentação e conforto.

Minamisako $^{18}$ (2015) elaborou um estudo comparativo de diferentes protocolos de laserterapia na ação analgésica e no reparo da mucosite oral radioinduzida associada ou não à quimioterapia. Foram avaliados 29 pacientes submetidos à radioterapia em região de cabeça e pescoço, divididos em 2 grupos (G1: laser vermelho na potência de $40 \mathrm{~mW}$; G2: combinação dos lasers vermelho e infravermelho na potência de $100 \mathrm{~mW}$ ). Não houve diferença significativa no efeito analgésico nos dois grupos avaliados.

A manutenção de boa higiene bucal, controle da xerostomia e tratamento de infecções oportunistas são essenciais para diminuir a severidade da mucosite, principalmente quando associados a suporte médico e nutricional adequado. Além do laser de baixa potência, diversas soluções para bochechos e medicamentos de ação tópica são úteis no controle da dor e da inflamação, atuando como coadjuvantes no tratamento ${ }^{19}$.

A cárie de radiação é um dos efeitos adversos do tratamento antineoplásico e está associada à hipofunção das glândulas salivares, promovendo xerostomia e subsequente alteração do pH bucal, que se torna mais ácido. As lesões cariosas se estabelecem em região cervical, deixam o esmalte opaco e a dentina enegrecida com consistência borrachoide, e podem levar à amputação da coroa dentária ${ }^{20}$. A paciente do referido estudo não apresentou até o momento lesões de cárie. 
BORGES BS, VALE DA, AOKI R, TRIVINO T, FERNANDES KS. Atendimento odontológico de paciente submetido à radioterapia em região de cabeça e pescoço: relato de caso clínico. Rev. Odontol. Univ. Cid. São Paulo 2018 jul/set 30(3) 332-40

ISSN 1983-5183

As medidas instituídas para prevenir a cárie de radiação incluem cuidados especiais como a higiene bucal, avaliações odontológicas periódicas e aplicação de flúor diário ${ }^{10}$. A paciente do estudo faz acompanhamento odontológico trimestral, uso diário de solução fluoretada e apresenta adequada higiene bucal.

Ciupa e Sá7 (2014) avaliaram 12 pacientes submetidos à radioterapia nas regiões de cabeça e pescoço e observaram que a xerostomia foi a manifestação bucal mais prevalente e debilitante nesses pacientes.

O tratamento da xerostomia inclui estimular o fluxo salivar por meio de gomas de mascar sem açúcar, uso de fluoretos em forma de gel ou solução, aumento da ingestão de líquidos, além do uso de substitutos salivares ${ }^{9}$. A paciente deste estudo ingere grande quantidade de líquidos diariamente e faz uso de lubrificantes bucais e labiais.

A osteorradionecrose (ORN) é caracterizada como um dos efeitos mais graves e tardios da radioterapia, sendo a mandíbula a estrutura mais afetada, devido à ação da radiação ionizante que causa hipóxia, hipovascularização e hipocelularização tecidual ${ }^{21}$.

Acredita-se que o risco de osteorradionecrose nesta paciente será baixo, pois ela realizou adequação bucal prévia à radioterapia e tem um acompanhamento odontológico intensivo. Pacientes que receberam tratamentos odontológicos previamente ao tratamento radioterápico, com intuito de adequação bucal e remoção de focos infecciosos, apresentam menos efeitos colaterais decorrentes da radioterapia ${ }^{21}$.

A prevenção da osteorradionecrose é essencial; dessa maneira deve-se realizar um planejamento minucioso antes do paciente iniciar a radioterapia e, nessa fase, é indicado extrair dentes com moderada a avançada doença periodontal, lesões periapicais extensas, lesões de cárie extensas, dentes parcialmente erupcionados, dentes impactados e raízes residuais. É recomendado que o intervalo entre a extração do dente e o início da radioterapia seja de 14 a 21 dias para se obter uma adequada cicatrização óssea ${ }^{13}$.

O tratamento da osteorradionecrose é realizado através da limpeza da ferida cirúrgica com soluções antimicrobianas, prescrição de bochechos de solução aquosa de gluconato de clorexidina a 0,12\% associado à higiene oral rigorosa, remoção de sequestros ósseos e possíveis irritantes locais²2.

Uma opção para o tratamento da oestorradionecrose é a oxigenação hiperbárica, na qual é empregando oxigênio sob alta pressão atmosférica, que promove o aumento de tensão de oxigênio na área comprometida, aumentando a atividade celular e o número de células, sendo, também, bactericida e bacteriostático ${ }^{22}$.

\section{CONCLUSÃO}

O acompanhamento odontológico do paciente irradiado em região de cabeça e pescoço antes, durante e após o tratamento antineoplásico, é fundamental para o controle e prevenção das complicações bucais, além de proporcionar aumento da qualidade de vida do paciente. 
BORGES BS, VALE DA, AOKI R, TRIVINO T, FERNANDES KS. Atendimento odontológico de paciente submetido à radioterapia em região de cabeça e pescoço: relato de caso clínico. Rev. Odontol. Univ. Cid. São Paulo 2018 jul/set 30(3) 332-40

ISSN 1983-5183

\section{REFERÊNCIAS}

1. JEMAL A, Siegel R, Ward E, Murray T, Xu J, Smigal C, et al. Cancer statistics, 2006. CA: a cancer journal for clinicians 2006 Mar-Apr;56(2):106-30.

2. JEMAL A, Siegel R, Xu J, Ward E. Cancer statistics, 2010. CA: a cancer journal for clinicians 2010 Sep-Oct;60(5):277-300.

3. INSTITUTO Nacional Do Câncer. INCA estima cerca de 600 mil casos novos de câncer para 2018. 2018 [Acesso em: 06 nov 2018]; Disponível em: http://www2.inca.gov.br/ wps/wcm/connect/agencianoticias/site/home/noticias/2018/inca-estima-cerca-600-mil-casos-novos-cancer-para-2018.

4. ARGIRIS A, Karamouzis MV, Raben D, Ferris RL. Head and neck cancer. Lancet (London, England) 2008 May 17;371(9625):1695-709.

5. COGNETTI DM, Weber RS, Lai SY. Head and neck cancer: an evolving treatment paradigm. Cancer 2008 Oct 1;113(7 Suppl):1911-32.

6. EPSTEIN JB, Thariat J, Bensadoun RJ, Barasch A, Murphy BA, Kolnick L, et al. Oral complications of cancer and cancer therapy: from cancer treatment to survivorship. $C A: a$ cancer journal for clinicians 2012 Nov-Dec;62(6):400-22.

7. CIUPA L, Sá ARN. Avaliação das complicações bucais em pacientes com câncer de cabeça e pescoço submetidos à radioterapia. Rev Saúde Biol 2014 9(2):4-12.

8. KATSURA K, Aoki K. Oral and dental healthcare for oral cancer patients: planning, management, and dental treatment. In: Kirita, T, Omura, K. Oral cancer: diagnosis and therapy. Tokyo: Springer Japan; 2015. p. 345-60.

9. ANDERSON L. Cuidados odontológicos em pacientes oncológicos. Onco\& 2014 set-out;25(1):24-6.

10. SANTOS CC, Noro-Filho GA, Caputo BV, Souza RC, Andrade DMR, Giovani EM. Condutas práticas e efetivas recomendadas ao cirurgião-dentista no tratamento pré, trans e pós do câncer bucal. J Health Sci Inst 2013 31(4):368-72.

11. TORRES SR, Gomes AOF. Protocolo clínico para cuidados odontológicos ao paciente submetido à radioterapia. CRO-RJ 2016 abril;3(28):1.

12. FLORENTINO ACA, Macedo DR, David EF, Carvalho K, Guedes CCFV. Tratamento da mucosite oral com laser de baixa potência: revisão sistemática de literatura. Rev Ciênc Méd, Campinas 2015 maio/ago;24(2):85-92.

13. DAVID EF, Ribeiro CV, Macedo DR, Florentino ACA, Guedes CCFV. Manejo terapêutico e preventivo da osteorradionecrose: revisão integrativa da literatura. Rev Bras Odontol 2016 jun.;73(2):150-6.

14. PAIVA MDEB, Biase RCCG, Moraes JJC, Ângelo AR, Honorato MCTM. Complicações orais decorrentes da terapia antineoplásica. Arq Odontol, Belo Horizonte 2010 mar;46(1):4855. 
ISSN 1983-5183

15. BONFIM GA, Guimarães EJ, Long SM, Ferreira MCD, Jóias RP. Mucosite oral em pacientes oncológicos. Odonto 2016 24(47):31-2.

16. GAETTI-JARDIM Júnior E, Sousa FRN, Gaetti-Jardim EC, Castro EVFL, Ciesielski FIN, Buso-Ramos MM, et al. Efeitos da radioterapia sobre as condições bucais de pacientes oncológicos. RPG rev pos-grad 2011 abr.-jun. ;18(2):96-101.

17. BUENO AC, Magalhães CS, Moreira AN. Associações entre fatores de risco e complicações bucais em pacientes com câncer de cabeça e pescoço tratados com radioterapia associada ou não à quimioterapia Pesqui bras odontopediatria clín integr 2012 jul.;12(2):187-93.

18. MINAMISAKO MC. Estudo comparativo de diferentes protocolos de laserterapia na ação analgésica e no reparo da mucosite oral radioinduzida associada ou não à quimioterapia [Dissertação]. Florianópolis: Universidade Federal de Santa Catarina, Centro de Ciências da Saúde, Programa de Pós-Graduação em Odontologia; 2015.

19. RIBEIRO Júnior O, Borba AM, Guimarães Júnior J. Prevenção e tratamento da mucosite oral: o papel fundamental do cirurgião-dentista. Rev Clín Pesq Odontol, Curitiba 2010 jan./abr. ;6(4):57-62.

20. FLORENTINO ACA, Oliveira CMAP, Carvalho K, Moura MAT, Faleiros CC, Guedes V. O papel da odontologia no pré, trans e pós-radioterapia em região de cabeça e pescoço, na prevenção e tratamento da cárie de radiação. Rev Universidade Vale do Rio Verde 2014 12(3):1.

21. GAETTI-JARDIM EC, Silva HCL, Pereira TTM, Faverani LP, Gonçalves JBO, Shinohara $\mathrm{EH}$, et al. Osteorradionecrose dos maxilares. Arch Health Invest 2014 3(6):37-40.

22. SANTOS R, Dall'magro AK, Giacobbo J, Lauxen JR, Dall'magro E. Osteorradionecrose em pacientes submetidos à radioterapia de cabeça e pescoço: relato de caso. RFO UPF 2015 ago.;20( 2):232-7.

RECEBIDO EM 07/03/2018

ACEITO EM 28/09/2018 